\title{
Calculating microbial growth rates from data on production and standing stocks
}

\author{
David L. Kirchman* \\ College of Marine Studies, University of Delaware, 700 Pilottown Road, Lewes, Delaware 19958, USA
}

\begin{abstract}
Growth rates of microbial assemblages are commonly estimated from data on biomass or cell abundance and rates of biomass or cell production. There are 2 algorithms for calculating growth rates from these data. One is to extrapolate from the measured standing stock $\left(B_{0}\right)$ to another standing stock level using the measured production rate. The growth rate is then calculated as $\ln \left[\left(P T+B_{0}\right) / B_{0}\right] / T$ where $P$ is the production rate and $T$ is a specific time interval which is $1 \mathrm{~d}$ when the growth rate is expressed with units of $\mathrm{d}^{-1}$. I argue here that this algorithm is conceptually incorrect and can underestimate the actual growth rate. The most appropriate approach is the simplest, that is, to divide the production rate by the estimate of biomass or cell abundance $(P / B)$. Additionally, propagation of errors analysis indicates that the uncertainty associated with growth rates estimates calculated from $P / B$ is lower than that calculated from $\ln \left[\left(P T+B_{0}\right) / B_{0}\right] / T$.
\end{abstract}

KEY WORDS: Exponential growth · Growth equations · Thymidine incorporation $\cdot$ Leucine incorporation

Resale or republication not permitted without written consent of the publisher

The growth rate is a fundamental property of all organisms and is especially informative about the activity of microbial populations that have the potential for increasing at exponential rates. Because of its importance, several methods have been used to estimate growth rates of various microbial groups in natural environments. Some methods, such as the frequency of dividing cells (Hagström et al. 1979) or cellular RNA content (Kemp et al. 1993), directly yield estimates of growth rates. Perhaps the most common approach, however, is to calculate growth rates from rates of cell or biomass production and cell abundance or biomass. This approach is used frequently to estimate growth rates of heterotrophic bacteria in aquatic environments (Ducklow 2000), but it is also used occasionally for phytoplankton as well (Smith et al 2000).

*E-mail: kirchman@udel.edu
The purpose of this note is to compare 2 different algorithms for calculating growth rates from production and cell abundance data. This note will focus on the mathematical basis of the calculations and will ignore all technical problems with estimating microbial production and biomass of natural assemblages as well as the conceptual difficulties in interpreting a growth rate for a mixed assemblage of microbes growing at different rates. The motivation behind this note is that some recent studies (see below) have used an approach to estimate growth rates that I will argue is conceptually incorrect. The most appropriate way to calculate growth rates is the simplest, that is, to divide the rate of cell production by an estimate of cell abundance.

Basic equations of exponential growth. The 2 algorithms to be discussed here share the same fundamental assumption: both treat the microbial community as a homogeneous population that would increase exponentially over time in the absence of mortality. Indeed, microbial standing stocks do not usually change substantially over time in natural environments because production is balanced by mortality. The degree of coupling between growth and mortality affects our interpretation of production and growth rate estimates, but not the calculation of growth rates from production and standing stock data. Regardless of this, mortality is ignored and exponential growth is assumed, in which case the change in microbial biomass over time $\left(\mathrm{d} B_{t} / \mathrm{d} t\right)$ is:

$$
\mathrm{d} B_{t} / \mathrm{d} t=\mu B_{t}
$$

where $B_{t}$ is the microbial biomass at time $t$ (units of cells or mass per unit volume, e.g. $\mu \mathrm{gC}^{-1}$ ) and $\mu$ the specific growth rate (units of per unit time, e.g. $\mathrm{d}^{-1}$ ). The well-known solution to Eq. (1) is: 


$$
B_{t}=B_{0} \mathrm{e}^{\mu t}
$$

where $B_{0}$ is the initial microbial biomass (i.e. $t=0$ ).

The key to my argument is to define production $(P)$ in terms of the parameters given in Eq. (1). Note that production is the increase in microbial biomass over time in the absence of any mortality and can be expressed mathematically as the slope of the curve on a graph of biomass versus time (not a semi-log graph). This slope is the first derivative of the biomass versus time curve. In short:

$$
P_{t}=\mathrm{d} B_{t} / \mathrm{d} t
$$

The subscript for time was added to $P$ in Eq. (3) to emphasize that $P$ changes with time because of changes in $B$, a critical point which will soon become clear. Note that $\mathrm{d} B_{t} / \mathrm{d} t$ has the same units as biomass production: biomass or cell abundance per unit volume per unit time. Given Eq. (3), then the growth rate is simply:

$$
\mu=\left(\mathrm{d} B_{t} / \mathrm{d} t\right) / B_{t}=P_{t} / B_{t}
$$

That is to say, growth rate is the rate of production divided by the standing stock.

An alternative algorithm to calculate growth rates. Several studies have used a different way to calculate growth rates, which seems quite reasonable. From Eq. (2), we can derive:

$$
\mu=\ln \left(B_{T} / B_{0}\right) / T
$$

where $T$ is the unit time interval; if $\mu$ is to have units of $\mathrm{d}^{-1}$ then $T$ must be $1 \mathrm{~d}$. Eq. (5) says that the growth rate is equivalent to the slope of a semi-log graph of biomass versus time, which is certainly true when standing stocks increase over time in the absence of mortality or more precisely when growth exceeds mortality and there is net growth. In any case, the standing stock data provide the estimate for $B_{0}$ and the production data are used to estimate $B_{T}$. It seems reasonable to assume that the change in biomass is the production multiplied by some time $(T)$ after the initial point (i.e. $t=0$ ). If this is indeed the case, then the biomass at this new time $T$ will be:

$$
B_{T}=P T+B_{0}
$$

I dropped the time subscript for $P$ in order to obtain equations similar to those used in previous studies . By substituting Eq. (6) into (5), we obtain the following equation for calculating the growth rate:

$$
\mu=\ln \left[\left(P T+B_{0}\right) / B_{0}\right] / T
$$

Since $\left(P T+B_{0}\right) / B_{0}=P T / B_{0}+B_{0} / B_{0}$, Eq. (7) is mathematically equivalent to:

$$
\mu=\ln \left(P / B_{0}+1\right)
$$

if the unit time interval is assumed to be $T=1$ (e.g. $1 \mathrm{~d}$ where production is expressed as mass per unit volume per d). Some early studies using this approach include Eppley \& Peterson (1978), who examined phytoplankton growth rates. More recent examples include Smith et al. (2000), who also examined phytoplankton and Sherr et al. (2001), who used thymidine and leucine incorporation to estimate growth rates of heterotrophic bacteria. It is worthwhile emphasizing that Eqs. (4), (7) \& (8) are all based on the same fundamental model of exponential microbial growth.

Illustration of the problem. The conceptual flaw in Eqs. (7) \& (8) lies in how the new biomass at time $=T$ is estimated. Eq. (6) gives the correct estimate of the growth rate only for $T \rightarrow 0$. Eq. (6) will yield erroneous growth rate estimates because $P$ is not a constant; as described in Eq. (3), production increases with time as standing stocks increase. In effect, Eq. (6) calculates the new biomass by a 1-step extrapolation from $t_{0}$ to $t_{T}$. This approximation is perhaps acceptable for small time intervals, but for sufficiently large values of $T$, the error of the extrapolation will impact the growth rate estimate. More precisely, the error could be substantial if the growth rate is large relative to $1 / T$.

The following hypothetical example illustrates the problem. Here, the initial microbial biomass is assumed to be $0.833 \mu \mathrm{MC}$, equivalent to $1 \times 10^{9}$ cells $^{-1}$ and $10 \mathrm{fgC} \mathrm{Cell}^{-1}$. The growth rate was then fixed at 1 , 0.5 or $0.1 \mathrm{~d}^{-1}$. Production at $t_{0}$ could then be estimated from graphs of biomass versus time or by simply using Eq. (1). Growth rates as a function of $T$ were calculated using Eq. (7).

The growth rate calculated by Eq. (7) decreases as $T$ increases regardless of the true growth rate (Fig. 1A). Consequently, the difference between the calculated and the true growth rate increases as $T$ increases (Fig. 1B). The error is not great for $T \ll 1$, and for low growth rates $\left(0.1 \mathrm{~d}^{-1}\right)$, but it quickly becomes substantial for faster growth rates. At $T=1 \mathrm{~d}$, the calculated growth rate is 19 and $30 \%$ too low when the actual growth rate is 0.5 and $1 \mathrm{~d}^{-1}$, respectively (Fig. 1B).

The preceding analysis applies only to the problem of estimating growth rates from standing stock and production data. When mortality has been minimized, it is still correct to estimate growth rates from the increase in bacterial abundance and biomass over time using a regression analysis, i.e. analogous to Eqs. (7) \& (8).

Propagation of errors. Another problem is that the uncertainty associated with the growth rate calculated by the 2 approaches differs; most of the time, the uncertainty will be larger for growth rates calculated with Eq. (7) or (8). With both algorithms, this uncertainty has to be calculated from the experimental errors associated with the standing stock and production measurements using appropriate propagation of error equations (Bevington 1969). In the case of Eq. (4) (i.e. $\mu=P / B)$, the uncertainty $\left(S_{\mu}\right)$ is: 


$$
S_{\mu}=\mu\left(S_{P}^{2} / P^{2}+S_{B}^{2} / B^{2}-2 S_{P B}^{2} / P^{2} B^{2}\right)^{0.5}
$$

where $S_{X}$ is the SE of the various parameters and $S_{P B}^{2}$ is the covariance of $P$ and $B$. For Eq. (7) or (8), the uncertainty is:

$$
S_{\mu}=\left(S_{P}{ }^{2} / P^{2}+S_{B}{ }^{2} / B^{2}-2 S_{P B}{ }^{2} / P^{2} B^{2}\right)^{0.5}
$$

Now the error calculated with Eq. (10) would seem to be smaller than that given by Eq. (9); however, exactly the opposite is usually the case since usually $\mu<1$. To illustrate this point, consider errors associated with the hypothetical case discussed above where $B=0.833 \mu \mathrm{MC}$, $P=0.417 \mu \mathrm{MC} \mathrm{d}^{-1}$ and $\mu=0.5 \mathrm{~d}^{-1}$. Assuming $S_{P B}{ }^{2}=0$ and that the errors for $P$ and $B$ are 10 and $20 \%$, respectively, the uncertainty associated with $\mu$ is 0.11 and $0.22 \mathrm{~d}^{-1}$ for

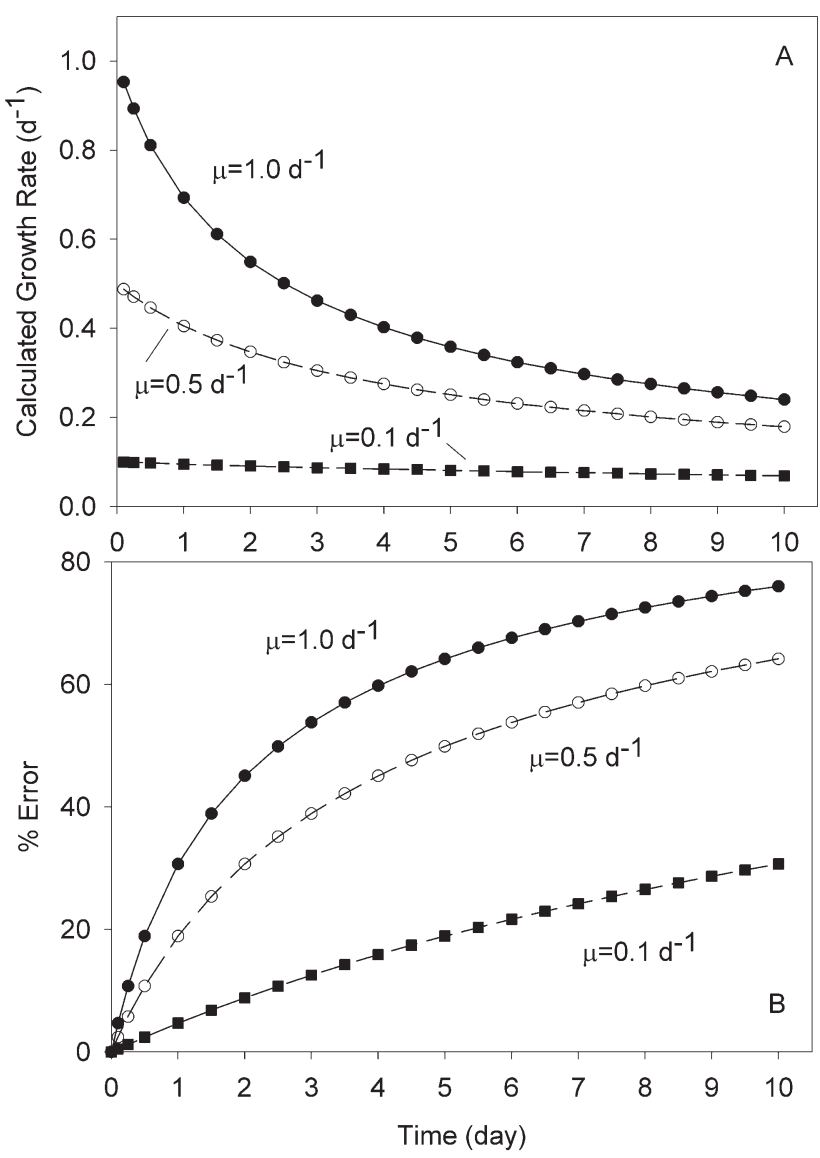

Fig. 1. Illustration of growth rates calculated from Eq. (7). (A) Growth rates calculated from Eq. (7) at various extrapolation times $(T)$ when the true growth rate is fixed at $1,0.5$ or $0.1 \mathrm{~d}^{-1}$. In essence, Eq. (7) uses the measured biomass production to extrapolate to a new biomass level at time $=T$. For this example, bacterial biomass is assumed to be $0.833 \mu \mathrm{MC}$, equivalent to $1 \times 10^{9}$ cells l$^{-1}$ and $10 \mathrm{fgC} \mathrm{cell}^{-1}$. (B) The difference in the growth rate calculated from Eq. (7) from the fixed growth rate. The percent error was calculated by dividing the growth rate calculated from Eq. (7) by the fixed growth rate, then multiplied by 100 the growth rate calculated by Eqs. (4) \& (8), respectively. In other words, according to the propagation of error analysis, the uncertainty of the growth rate calculated by Eq. (8) is twice that of Eq. (4) $(\mu=P / B)$ for these particular values of $P, B$ and the growth rate.

Concluding remarks. The preceding discussion also applies to interpreting the ratio of production rates to cell abundance. Given the unknowns in estimating bacterial biomass and production, some investigators report, for example, incorporation rates of leucine per cell rather than growth rates (e.g. Kirchman et al. 2000). This ratio can be used as a proxy for growth rates if it is agreed that Eq. (4) is the correct algorithm for calculating growth rates. If Eq. (7) or (8) is thought to be the correct algorithm, the relationship between growth rate and incorporation rates per cell becomes unclear. In contrast, if $\mu=P / B$, then there is a direct relationship between incorporation rates per cell and growth rates.

The errors from using Eqs. (7) \& (8) pale in comparison to the many problems in estimating bacterial biomass and biomass production, and the conceptual difficulties in interpreting a growth rate for a mixed microbial assemblage. Additionally, it could be argued that the difference in growth rates calculated by the 2 algorithms discussed here are usually trivial for most applications, i.e. when the incubation time is short compared to the generation time of the microbial assemblage. However, even if we ignore the large difference in the propagation of errors associated with the 2 algorithms (2-fold in the example given above), there is no reason to use the wrong algorithm. For once, the simplest algorithm with the lowest calculated uncertainty is the most appropriate.

Acknowledgements. I thank Hugh Ducklow for commenting on an early draft of this paper. This work was supported by grants from the National Science Foundation.

\section{LITERATURE CITED}

Bevington PR (1969) Data reduction and error analysis for the physical sciences. McGraw Hill, New York

Ducklow H (2000) Bacterial production and biomass in the oceans. In: Kirchman DL (ed) Microbial ecology of the oceans. Wiley-Liss, New York

Eppley RW (1968) An incubation method for estimating the carbon content of phytoplankton in natural samples. Limnol Oceanogr 13:574-582

Hagström ^̊, Larsson U, Horstedt P, Normark S (1979) Frequency of dividing cells, a new approach to the determination of bacterial growth rates in aquatic environments. Appl Environ Microbiol 37:805-812

Kemp PF, Lee S, Laroche J (1993) Estimating the growth rate of slowly growing marine bacteria from RNA content. Appl Environ Microbiol 59:2594-2601 
Kirchman DL, Meon B, Cottrell MT, Hutchins DA, Weeks D, Bruland KW (2000) Carbon versus iron limitation of bacterial growth in the California upwelling regime. Limnol Oceanogr 45:1681-1688

Peterson BJ (1978) Radiocarbon uptake: its relation to net particulate carbon production. Limnol Oceanogr 23: 179-184

Editorial responsibility: Otto Kinne (Editor),

Oldendorf/Luhe, Germany
Sherr EB, Sherr BF, Cowles TJ (2001) Mesoscale variability in bacterial activity in the Northeast Pacific Ocean off Oregon, USA. Aquat Microb Ecol 25:21-30

Smith WO, Marra J, Hiscock MR, Barber RT (2000) The seasonal cycle of phytoplankton biomass and primary productivity in the Ross Sea, Antarctica. Deep-Sea Res Part II $47: 3119-3140$

Submitted: October 24, 2001; Accepted: February 28, 2002 Proofs received from author(s): April 17, 2002 